Revue pluridisciplinaire du monde antique

18 | 2002

Le statut et l'image du corps dans la mythologie et la littérature grecques

\title{
Comment inventer un corps différent
}

\section{Lucian Boia}

\section{(2) OpenEdition \\ Journals}

Édition électronique

URL : http://journals.openedition.org/kentron/1930

DOI : $10.4000 /$ kentron. 1930

ISSN : 2264-1459

Éditeur

Presses universitaires de Caen

\section{Édition imprimée}

Date de publication : 31 décembre 2002

Pagination : 31-35

ISBN : 2-84133-202-0

ISSN : 0765-0590

Référence électronique

Lucian Boia, "Comment inventer un corps différent », Kentron [En ligne], 18| 2002, mis en ligne le 01 octobre 2018, consulté le 21 novembre 2020. URL : http://journals.openedition.org/kentron/1930 ; DOI : https://doi.org/10.4000/kentron.1930

\section{(c) (i) (9)}

Kentron is licensed under a Creative Commons Attribution-NonCommercial-NoDerivatives 3.0 International License. 


\section{COMMENT INVENTER UN CORPS DIFFÉRENT ${ }^{1}$}

Réalisable ou non (l'avenir le dira), la transformation - voire la transfiguration - du corps humain est un projet qui appartient de plein droit à l'imaginaire. Un projet tenace qui vient de loin, un rêve quasi permanent de l'humanité.

Les études sur l'imaginaire sont tiraillées entre deux tendances. Certains théoriciens privilégient les structures durables (essentiellement les mêmes à travers les époques et les cultures), tandis que les autres insistent sur le mouvement, sur un imaginaire toujours différent, adapté au contexte historique. Un philosophe anthropologue comme Gilbert Durand représente la première tendance, tandis que la seconde est illustrée par Jacques Le Goff, un historien. Personnellement, je crois que les deux attitudes sont plutôt complémentaires qu’opposées. La dialectique de l'imaginaire réunit les permanences et le changement. L'homme est toujours le même et il est toujours différent.

Il me semble qu'une des structures les plus caractéristiques de l'esprit humain est celle qu'on pourrait définir par le désir d'évasion. Sortir de l'histoire, sortir du temps, sortir de sa propre condition, voilà une des obsessions de l'humanité. Il s'agit de reformuler radicalement la condition humaine, par l'invention d'un monde différent et d'un être humain différent.

La méthodologie d'une telle démarche présente une certaine variété. On peut regarder vers les commencements (âge d'or, paradis perdu), vers des îles lointaines ou atemporelles (utopies) ou vers l'avenir (du millénarisme religieux à «l'avenir radieux» des idéologies modernes). L'être humain peut devenir différent par progression ou par régression, s'envolant vers le ciel ou se réintégrant dans la nature, dans les variantes opposées d' "homme-dieu» ou "homme-bête» (ou dans une formule synthétique réunissant ces deux extrêmes).

L'Antiquité connaissait déjà toutes les formules. À l'époque, les hommes très différents se trouvaient vers les marges du monde. Dans les régions lointaines de la Scythie vivaient soit des cannibales féroces, soit, par contre, des végétariens justes et sages, presque des saints. L'Inde (figurant à la fois l'extrémité orientale et méridionale

1. Communication prononcée le mercredi 18 octobre 2000. 
de l'œecoumène) présentait une variété inépuisable d'hommes différents: hommesbêtes (comme les cynocéphales, êtres «humains » à tête de chien), hommes primitifs (pygmées) ou des êtres humains très spiritualisés, « dématérialisés » en quelque sorte (le cas des brahmanes; d'ailleurs, selon les dires de Ctésias, les Indiens généralement étaient " très justes »).

Mais, d'autre part, même dans la cité, à l'intérieur d'une communauté normale, il y avait des méthodes susceptibles de modifier l'être humain. En Grèce, le culte de Dionysos (et son expression philosophique, le cynisme) se proposait de rapprocher l'homme de l'état de nature; par contre, le mouvement orphique et le pythagorisme misaient sur les valeurs spirituelles, cherchant à rapprocher l'homme des dieux. En Chine, le taoïsme préconisait une purification spirituelle, le détachement de la matière - ce qui menait aussi à la longévité, voire à une quasi-immortalité (une "espérance de vie» de mille ans, peut-être même de dix mille). Le christianisme se proposa à son tour de changer la nature spirituelle de l'homme, de le purifier et de lui ouvrir la voie de la transcendance. "L'homme nouveau ", syntagme repris plus tard par les totalitarismes, fut à l'origine un concept chrétien.

Ainsi, on peut constater aisément que la modernité n'a fait que reprendre des clichés et des projets bien anciens. Elle les a tout simplement sécularisés et obligés à se soumettre aux nouvelles idéologies (de cette manière, le perfectionnement de l'homme sera assuré soit par la démocratie de type libéral, soit par le communisme, ou le nazisme...). Elle les a liés aussi aux sciences et aux technologies nouvelles, et tout particulièrement à la conception évolutionniste qui s'est imposée à partir de Lamarck et de Darwin. Grâce à la science, l'homme réussira à transformer le monde et finira par se transformer lui-même. Le mot clef qui réunit les projets de l'âge moderne est le Progrès.

Pour Condorcet, dans son Esquisse d'un tableau historique des progrès de l'esprit humain (écrite en 1793 et publiée en 1795, texte fondateur pour la quête moderne de l'avenir), la société nouvelle, libérée des anciennes inégalités et injustices, fera naître, logiquement, un être humain nouveau: plus fort, plus sain et plus intelligent que son ancêtre, et ayant en plus la chance de vivre plus longtemps, l'espérance de vie devant augmenter indéfiniment.

C'est un projet auquel le XIX siècle croit fermement: on est en pleine époque d'optimisme scientifique. Voici, par exemple, Renan, dans ses Dialogues philosophiques (1871):

Une large application des découvertes de la physiologie et du principe de sélection pourrait amener la création d'une race supérieure, ayant son droit de gouverner, non seulement dans sa science, mais dans la supériorité même de son sang, de son cerveau, et de ses nerfs... La nature jusqu'ici a fait ce qu'elle a pu... C'est à la science à prendre l'œuvre au point où la nature l'a laissée... De même que l'humanité est sortie de l'animalité, ainsi la divinité sortirait de l'humanité. 
L'évolution permettait tous les espoirs; elle justifiait aussi toutes les craintes. L'avenir débouchait sur une variété de scénarios. Ce n'était qu'une question de temps, de durée. L'Homme de l'an 1000000 est le titre d'un essai publié par H.G. Wells en 1893 ; notre lointain descendant apparaît comme une sorte de pieuvre, avec des tentacules, un corps frêle, presque inexistant (diminution des fonctions proprement animales), mais, en revanche, une tête immense (développement de l'intelligence). Toujours Wells a imaginé, dans La Machine à explorer le temps (1895), une bifurcation de l'humanité conduisant à l'apparition de deux espèces également repoussantes, les féroces Morlocks et les Eloïs imbéciles, les premiers descendant des prolétaires et les seconds de la classe dominante (projection dans l'an 802701 de la société polarisée du XIX ${ }^{e}$ siècle).

Ce genre d'imaginaire, appliqué à l'avenir terrestre, est encore plus présent sur les planètes qui figurent, selon l'évolutionnisme du XIX siècle, des phases différentes de notre propre histoire ou de l'histoire de la Terre. Mars, planète plus «âgée » que la Terre, offre généralement les scénarios de l'avenir, tandis que Vénus, plus jeune, abrite une humanité préhistorique dans un décor qui réactualise les époques géologiques révolues. Vers 1900, Mars est la vedette incontestée de l'espace. Ses habitants, d'une variété corporelle déroutante : géants ou nains, "grosses têtes", hommes ailés..., sont le plus souvent supérieurs aux hommes terrestres. Ce fut à l'origine du mythe actuel des extraterrestres à la seule différence que ceux-ci, de nos jours, ne viennent plus des planètes proches mais des profondeurs des galaxies. Comme les Martiens d'hier, les extraterrestres d'aujourd'hui se remarquent par une évolution biologique avancée et non moins par leur technologie sophistiquée. À l'autre extrême se trouve l'homme sauvage, apparemment toujours présent, sur la Terre même, dans certaines régions peu abordables; le yéti est la figure la plus populaire de cette espèce. L'homme - notre propre espèce - évolue à mi-chemin entre ces deux extrêmes, suivant une disposition qui, mutatis mutandis, reste essentiellement la même que celle imaginée dans l'Antiquité (une humanité qui se tient à égale distance de l'" animalité » et de la «divinité », partageant partiellement leur substance mais gardant toutefois sa spécificité proprement humaine).

Laisser l'évolution faire son travail (pour un avenir lointain) ou attendre l'arrivée des extraterrestres ne semblait pas du goût de tout le monde. Le XIx ${ }^{e}$ siècle fut à la fois déterministe et volontariste. L'évolution était considérée comme un processus objectif et «déterminé» mais, en connaissant ses lois, on pouvait la contrôler, la canaliser, l'accélérer... Ainsi naquit l'eugénisme (avec les travaux de Francis Galton: Hereditary Genius, 1869; Natural Inheritance, 1889). Pour les adeptes de cette nouvelle science, il s'agissait en premier lieu de surveiller la reproduction afin d'encourager les unions bénéfiques et de décourager la "perpétuation » des exemplaires « dégénérés » de l'espèce humaine. Alexis Carrel, prix Nobel de médecine, s'exprime très clairement sur ce sujet dans son livre L'Homme, cet inconnu (1936), un best-seller à l'époque : 
La science, qui a transformé le monde matériel, nous donne le pouvoir de nous transformer nous-mêmes... Il y a un seul moyen d'empêcher la prédominance désastreuse des faibles. C’est de développer les forts.

Les « autres» sont une quantité négligeable. Pour les exemplaires les moins réussis ou les plus pervertis, Carrel recommande carrément la chambre à gaz. Théorie élitiste, l'eugénisme a fait carrière en Allemagne nazie, mais aussi dans les pays « démocratiques» ( $\mathrm{y}$ compris en France, au moins au niveau théorique), à une époque où l'inégalité sociale et biologique était une chose qui allait de soi. Certaines méthodes dures - castration, stérilisation des fous, des criminels, des personnes dégénérées (concept particulièrement élastique!) - furent appliquées même dans des pays comme les États-Unis et la Suède.

Le concept communiste de l' «homme nouveau » a été apparemment plus démocratique et plus généreux. La fin de «l'exploitation de l'homme par l'homme» devait permettre l'épanouissement du corps et de l'esprit humains. Afin d'y aboutir, le communisme, idéologie "prolétarienne », préconisait en premier lieu la méthodologie du travail: le travail qui, d'après Engels, avait transformé le singe en homme, transformera l'homme en «homme nouveau ». Associée au travail, la vie en collectivité était le deuxième grand principe. «Travail» et « collectivité» résument la pédagogie de Makarenko qui a formé pendant des dizaines d'années le fondement du système éducatif de l'Union soviétique et des autres pays communistes. Pour l'avenir - un avenir proche - on attendait des merveilles : l'homme communiste devait devenir de plus en plus fort, de plus en plus sain, de plus en plus efficace; son espérance de vie devait augmenter considérablement (jusqu'à 150 ans, selon le docteur Bogomoletz - voir son livre Comment prolonger la vie?, publié en 1938 et traduit en français en 1950. D’ailleurs les habitants du Caucase - pays de Staline ! - donnaient déjà le bon exemple, certains prolongeant leur vie - au moins dans l'imaginaire - jusqu'à un siècle et demi et même au-delà). Les pays communistes impressionnaient déjà le monde par la multitude de leurs records sportifs, ce qui semblait indiquer que la fabrication d'un être humain plus performant était en bonne voie. Malheureusement, l'explication de ce phénomène est moins exaltante : l'État communiste élevait soigneusement (presque au sens propre du mot "élevage») les sportifs de performance pour des motifs évidents de prestige. Le principe tant proclamé de l'épanouissement de l'homme devint en fait un nouvel esclavage. Loin de connaître un perfectionnement continu, le corps humain a été agressé et finalement dégradé par les dictatures communistes. Conditions pénibles de travail, nourriture médiocre, assistance médicale insuffisante, dérèglements de la vie quotidienne - tout a contribué à un état de santé qui est loin d'être brillant. L'espérance de vie en fournit un indicateur incontestable; en dépit des promesses mirobolantes, elle se trouve aujourd'hui dans l'espace ex-communiste à un niveau 
sensiblement plus bas que dans l'Occident «capitaliste» (presque une dizaine d'années de moins!).

L'expérience communiste a échoué. Et le projet eugénique n'est évidemment pas satisfaisant, vu ses insuffisances scientifiques et ses préjugés sociaux. Mais l'objectif d'améliorer l'espèce humaine n'est pas abandonné pour autant. Par contre, il semble se trouver à la veille d'une nouvelle offensive. Ses instruments scientifiques se sont beaucoup affinés. Actuellement c'est la génétique qui mène le jeu; le déchiffrement du génome humain et la technique du clonage ouvrent des perspectives apparemment sans limites. Ceci suscite à la fois des espérances et des craintes. Jusqu'où peut-on aller, jusqu'où a-t-on le droit d'aller? Est-ce qu'un changement radical de la condition humaine serait possible? Et s'il était possible, serait-il aussi souhaitable?

La santé est devenue l'objectif prioritaire de la civilisation occidentale (presque une religion, occupant le terrain laissé libre par le reflux des croyances religieuses et des idéologies; de plus en plus, l'individu entend vivre pleinement sa vie plutôt que de l'investir dans un hypothétique au-delà ou dans des projets strictement collectifs). La maladie est ressentie comme un scandale, et la mort aussi (ou presque). La campagne antimaladie est déjà lancée et elle ira sans doute en s'amplifiant. Il en résulte tout un faisceau de scénarios: des plus modérés (une amélioration à l'intérieur de certaines limites considérées comme naturelles) aux plus audacieux (dépassement de toutes les limites; ainsi, une espérance de vie étalée sur des centaines d'années !). Mais l'avenir est un terrain inconnu. À coup sûr, il sera différent de tous nos scénarios.

Lucian BoIA

Université de Bucarest

\section{Notice bibliographique}

Ce texte présente d'une manière synthétique un sujet que j'ai développé dans plusieurs de mes livres, et notamment dans L'Exploration imaginaire de l'espace, Paris, La Découverte, 1987 ; Entre l'Ange et la Bête. Le mythe de l'Homme différent de l'Antiquité à nos jours, Paris, Plon, 1995; et La Mythologie scientifique du communisme, Paris, Les Belles Lettres, 2000.

Pour les tendances récentes, voir l'ouvrage de Lucien Sfez, La Santé parfaite. Critique d'une nouvelle utopie, Paris, Seuil, 1995, et, sous sa direction, L'Utopie de la santé parfaite (Colloque de Cerisy-la-Salle, 11-19 juin 1998), Paris, PUF, 2001. Des scénarios pour un avenir relativement proche, chez Michio Kaku, Visions. Comment la science va révolutionner le Xxi siècle, Paris, Albin Michel, 1999.

L.B. 